\title{
Andere Helden: \\ Morphologien des Heroisch-Fremden im Tanztheater des 19. Jahrhunderts
}

\section{Claudia Jeschke / Gabi Vettermann}

Das fremde Heroische zeigt sich auf den Ballettbühnen im 19. Jahrhundert als fragmentiertes, deformiertes Heldentum. Fremde, Helden und Heldinnen, fremde Helden und Heldinnen sind zwar präsente, wenn auch uneindeutige, komplexe Figuren - in doppelter Hinsicht: Auf der Ebene der Personalfiguration als auch im Umgang mit traditionellen Strukturen tänzerischer Inszenierung verweisen sie zunächst auf das Entwurfspotenzial des Fremden allgemein. ${ }^{1}$ Gleichermaßen und über das theatrale Möglichkeitsspektrum des Fremden hinaus intensivieren oder stören fremde Helden und Heldinnen die Auseinandersetzung mit zeittypischen szenischen Qualitäten durch Kollusion und Kollision: Indem sie Fragmente von heldischem Außeralltäglichem, Exzeptionellem affirmativ beziehungsweise deformativ verkörpern, explorieren sie (tanztheater-relevante) ästhetische und strukturelle Verschiebungen und Morphologien des Heroischen. ${ }^{2}$ Anhand dreier Beispiele soll diese Verstörung durch fremde Helden deutlich gemacht werden: Danina, oder Joko, der brasilische Affe, (1826/1830), Quasimodo ou La Bohémienne (1859) und La Péri (1842/1843).

Ein Charakteristikum des Fremden, des fremden Heroischen im Tanztheater des 19. Jahrhunderts - so die These - ist, dass es vor allem paarweise funktioniert, wobei das ,Paar', die ProtagonistInnen, vor dem Hintergrund einer erkennbaren, definitiven Gemeinschaft wie Familie, Hofstaat, Bürgertum agiert. Einer der beiden stammt von ,Anderswo ${ }^{63}$ - so wird Danina als Brasilianerin eingeführt, La Bohémienne, Esmeralda, ist Zigeunerin und Achmet, der im Titel des Balletts nicht genannte zweite Protagonist des Balletts La Péri, firmiert als ,Orientale‘. Die anderen Personen der Zweierbeziehung sind Fremde im Sinn von Außenseitern - der

1 Claudia Jeschke [et al.]: Interaktion und Rhythmus. Zur Modellierung von Fremdheit im Tanztheater des 19. Jabrbunderts. München 2010, 516: „Indem das Tanztheater der ersten Hälfte des 19. Jahrhunderts das Fremde als Modell für das Überdenken wie Ausprobieren von Aufführungspraktiken einsetzt, wird Fremdheit zum Generator szenischer und narrativer Möglichkeiten, der choreographischen Transgression und des tänzerischen Überflusses.“

2 Die folgende Untersuchung perspektiviert (fremde) ProtagonistInnen des Ballettepertoires nicht nur als zentrale, sondern als offensichtlich auch mit heroisch-affinen Funktionen und Aktionen ausgestattete Bühnenfiguren. Der Argumentation dieses Artikels liegt demnach ein weniger normativer als vielmehr de-essenzialisierter und relationaler Umgang mit dem Begriff des Heroischem zugrunde.

3 Bernhard Waldenfels: Studien zur Phänomenologie des Fremden, Bd. 1: Topographie des Fremden. Frankfurt am Main 1997, 123: „[Das Fremde] entstammt einem unwiderruflichen Einst und Anderswo." 
anthropomorphe Joko, der physisch amorphe Quasimodo und die metamorphe Péri.

Fremd-heroische Phänomene sind in den hier zu verhandelnden tanztheatralen Beispielen also weder schwarz noch weiß, gut oder böse, natürlich wild oder gezähmt kulturell, menschlich oder kreatürlich, sondern zeigen diese und andere Eigenschaften - unabhängig vom Geschlecht - jeweils verschieden in den szenischen Interaktionen mit ihrem Partner beziehungsweise ihrer Partnerin und weiter durch den (nur?) handwerklich-traditionellen oder (provokant?) virtuosen Umgang mit Körper und Bewegung, Physis und Körperumraum, Körper- und Bühnenraum beziehungsweise tanztheatraler Aufführungsgeschichte(n).

Die These von der Uneindeutigkeit oder Komplexität von fremdem Heldentum gründet auf einer spezifischen, an der Tanzpraxis orientierten Lektüre der Quellen: Libretti als verbalschriftliche Dokumente und Inszenierungsnotate als grafische Dokumente mit verbalen Erläuterungen lassen sich jenseits ihrer Narrativik - auch - in Bezug auf ihr performatorisches Potenzial interpretieren; unter dieser Perspektive verweisen sie auf, tatsächliche ${ }^{6}$ Optionen von Aufführen oder Durchführen. Sie sind Handlungsweisungen, reflektieren die Tanzbarkeit von Narration ebenso wie die Narrativik des Tanzes. Es ist der Blick auf das performatorische Potenzial, der dem Fremden/Heroischen ein, so zeigt sich, dramaturgisch und choreografisch verstörendes wie störendes Rollen- und Handlungsspektrum eröffnet.

\section{Heroisch-Fremde als Spielmacher: \\ Danina, oder Joko, der brasilische Affe $(1826 / 1830)^{4}$}

Kurz zum Inhalt des Balletts: Die indigene Fremde Danina hat heimlich einen Sohn, Zabi, mit dem Kolonial-Fremden Don Alvaro. Die Heimkehr des abwesenden Alvaros drängt das Paar zur Offenbarung oder Legalisierung ihrer Beziehung. Als obligatorische Gegenspieler fungieren Don Alonso, Alvaros Vater, und ein Konkurrent um Daninas Gunst namens Jeauffre. Sie gilt es zu überzeugen, zu ,besiegen', oder anders: die Fremd-Eingeborenen und die Fremd-Kolonialisten müssen sich versöhnen. ${ }^{5}$

4 Der Evaluierung liegen zwei Libretti zugrunde, die 1826er und 1830er Fassung: Peter Joseph von Lindpaintner, Filippo Taglioni, Antoine Titus: Danina, oder: Joko der brasilische Affe. Ballett in 3 Abtheilungen. Berlin 1826. Peter Joseph von Lindpaintner, Filippo Taglioni, Weidner: Danina, oder: Joko der brasilianische Affe. Idealisches Ballett in 4 Akten. [1] [ca. 1830].

5 Das Ballett ,funktioniert' beziehungsweise hat zu funktionieren, egal ob es sich bei dem ,Vertreter der Kolonien' um einen Pflanzer, seinen Sohn (ca. 1830) oder (in der 1826er Fassung) um den Gouverneur einer Provinz von Brasilien, den Kommandeur der portugiesischen Flotte (im Laufe der Handlung als Sohn des Gouverneurs erkennbar) handelt. Ähnlich austauschbar ist das (ethnische und soziale) Profil des ,Mitbewerbers" um Danina, einen ,Mulatten', Beamter der Verwaltung und Daninas Freier (1826) oder einen ,Mohren', ebenso Daninas Freier beziehungsweise der Gegenspieler des Kommandanten oder Pflanzersohns (ca. 1830). Und die Personenfiguration unterscheidet auch nicht zwischen freien 
Der Aktionsspielraum der Rollenfigur des Affen als eines erkennbaren, physisch präsenten Nicht-Menschen bietet die Möglichkeit, diesen Inhalt zu fokussieren, und die Handlung voranzutreiben. Unter dem Blickwinkel der Tanz- und Theatergeschichte bestätigt die körperliche, gestische Qualität der Affendarstellung die theatralen Präsentationsmodi der Groteske: Jokos Auftritte und Handlungen haben Ähnlichkeiten mit den traditionellem Darstellungen des ,guten Negers ${ }^{6}$ in Balletten des 18. und zu Beginn des 19. Jahrhunderts. ${ }^{7}$ Gleichzeitig eröffnen sie einen performativen Freiraum, das heißt den für den Affen als befremdlichen Titelhelden notwendigen Modifikationsradius. Diese dem zeitgenössischen Publikum vermutlich mehr oder weniger bekannten showe-acts a-menschlicher Verhaltensweisen lassen ihn vertraut, komisch, ,menschlich' erscheinen.

Wie brüchig diese überkommene Darstellung jedoch ist, zeigt sich besonders deutlich in Jokos irritierenden und notwendigerweise freien Interaktionen gegen Ende des Balletts, wenn die Versöhnung zwischen indigenen und kolonialen Fremden herbeigeführt werden muss: Die bewegungsorientierte Ungebundenheit, die Anarchie des anthropomorphen Helden als Spielmacher reicht nicht aus, um die Handlung voranzubringen. Eine narrative Option wird benötigt: Um die Rettung des Sohnes Zabi durch Joko, den Affen, zu vermitteln, wird diese Rettung in der jüngeren Fassung vom Sohn nacherzählt. Die rasante Abfolge von Aktionen, zusätzlich noch befördert durch ihre Ausgangssituation, eine festliche Idylle mit Tanz der (heimlichen) Familie, war wohl nicht nur physisch, gestisch, choreografisch zu vermitteln. Oder, so ließe sich spekulieren, die drolligen Darbietungen des Affen standen zu sehr im Vordergrund und lenkten von der Handlung ab. ${ }^{8}$

Die Funktion von Danina, der ,anderen' fremden Heldin des Balletts, zeigt sich unter anderem in der getanzten Festszene. Feste sind ein tradiertes Mittel des Balletts, denn auf Festen wird getanzt, was üblicherweise heißt, dass der dramaturgisch begründete Fortgang der Geschichte angehalten wird. Das Fest, das Tanzen erhält nun in diesem Ballett eine historisch ungewohnte, irritierende Qualität, ist nicht mehr selbstverständlich, sondern muss argumentativ konterkariert werden, um in

Pflanzern oder Angestellten des Gouverneurs oder Sklaven. Identifizierbar sind die Figuren durch ihre Namen, Alonzo oder Don Alonso (der Vater), Don Alvar oder Alvaro (der Sohn), die weniger charakterisieren, als dass sie als couleur locale eingesetzt werden.

6 Joellen A. Meglin: „Sauvages, Sex Roles, and Semiotics“: Representations of Native Americans in the French Ballet, 1736-1837, Part One: The Eighteenth Century. In: Dance Chronicle 23: 2, 2000, 87-132; Part Two: The Nineteenth Century. In: Dance Chronicle 23: 3, 2000, 275-320.

7 Slapstickeinlagen ziehen sich durch das gesamte Ballett. So etwa zu Beginn, wenn Joko vor der Schlange flieht, im II. Akt, 4. und 5. Szene, wenn er mit Hut und Mantel einen Menschen mimt und auch als solcher (v)erkannt wird, die Situation aber ausnutzt, um ganz ,unschuldig' Obst zu stibitzen. Oder sie werden erkennbar in seinen vielfältigen Aktionen mit Musikinstrumenten.

8 In der 1826er, der kürzeren Fassung nur angedeutet, wird eine ganze Szene verwendet, um den possierlichen Umgang von Affe und Kind zu zeigen. Joko setzt alle körperlichen Mittel ein, um dem Jungen spielerisch nahezukommen. Ihrer beider Interaktionen werden zum unterhaltsamen Display. 
der bislang unabgeschlossenen Story zu greifen: Grund der Feier ist die ,Heimkehr Don Alvaros, des heimlichen Mannes von Danina und Vaters von Zabi, die jedoch überschattet ist von der drohenden (und im Schlussakt schließlich vollzogenen) Offenbarung der bislang versteckten Familie durch den Gegenspieler Jeauffre. ${ }^{9} \mathrm{Da}-$ nina organisiert und dirigiert das Fest - als indigene Brasilianerin eine Fremde, die nicht zur herrschenden Klasse gehört und dennoch heimlich mit ihr verbunden ist durch Mann und Kind. In der hier praktizierten Engführung von tänzerischem und interaktivem Blickwinkel setzen sich Handlung und Tanzen leichtfüßig und temporeich über mögliche Ungereimtheiten hinweg. Die spätere Librettofassung benennt die verwendeten National-, das heißt fremden Tänze und zitiert damit die zusätzliche performative Dimension fremd-differenter, tänzerischer Mentalitäten. Das Tanz-Fest dient trotz oder gerade wegen seiner unüblichen immanenten Dramatik zur Präsentation aller virtuosen tänzerischen Fähigkeiten - ein Verweis auf die direkte dramaturgische und indirekte choreografische Funktion der Rolle Daninas, die, ebenso wie die Figur des Affen, auf die fragmentierende, deformierende Bedeutung von Physis und Bewegung für die Darstellung von heroisch Fremdem hindeutet.

Die notwendige oder argumentativ vorgestellte Auseinandersetzung mit dem/ den Fremden extrapoliert die Verbindung von Alterität und Heroischem als verkörperte, also physisch-mimische, wie tänzerische Charakteristika und gleichzeitig als irritierende Integrationsmöglichkeiten. Danina und Joko werden unterschiedliche, jedoch immer mobile Strategien zugewiesen, wie sie traditionelle Personenfigurationen verlassen und unerwartete Aktionsmöglichkeiten entdecken oder sich diesen auch wieder anpassen. Dies geschieht durch ihre Interaktionen als Paar, das diese Mobilität zu stimulieren und zu regulieren scheint und somit dramaturgische wie choreografische Traditionsmuster infrage stellt.

\section{Das deformierte Heroische: \\ Quasimodo ou La Bohémienne (1859)10}

Kurz zum Inhalt des Balletts: Anlässlich eines öffentlichen Festes versucht der Dichter Pierre Gringoire vergebens sein Stück aufzuführen. Das Publikum will kein Mysterienspiel sehen, sondern unterhalten werden. Unterhaltung findet es im zum ,Narrenpapst‘ gekürten Quasimodo, dem Glöckner von Notre-Dame,

9 Ein traditionell übliches, das heißt ungetrübtes Fest kann denn auch erst nach der Eingliederung der Familie in die Gesellschaft gefeiert werden - nachdem Alonzo den Segen zur Heirat beziehungsweise zum Kind gegeben hat. In der 1826er Fassung sind die Festlichkeiten in den III. Akt integriert; die spätere Fassung widmet dem Tanz-Fest einen Extra-, den IV. Akt.

10 Ouasimodo ou La Bohémienne. Ballet d'actions en trois actes et six tableaux. Par M. Justamant. Musique de M. Luigini, Décors de M. Devoir, Costumes de M. Blod. Mise en Scène et notes détaillés sur tout a que contient l'ouvrage. Representé pour la $1^{\text {re }}$ fois sur le grand théatre a Lyon 1859 (Schreibweise entspricht dem Manuskript). 
und in den Tänzen der Zigeunerin Esmeralda. Während des Tanzes kommt es zur Begegnung mit dem Archidiakon Claude Frollo, der in Esmeralda verliebt ist. Sie aber meidet ihn. Frollo und sein Diener/Ziehsohn versuchen, Esmeralda zu entführen. Sie wird jedoch von Phœbus, dem General der Königlichen Bogenschützen, befreit und verliebt sich in ihn. Im Cour des Miracles rettet Esmeralda den Dichter Gringoire vor dem Tod, indem sie ihn heiratet. Esmeralda zeigt ihr tänzerisches Können auf einem Fest und entdeckt, dass dessen Anlass die Verlobung ihres ,Geliebten' Phœbus mit Fleur de Lys ist. Esmeralda und Phœbus treffen sich; bei diesem Rendezvous verübt Frollo einen Mordanschlag auf seinen Nebenbuhler. Frollo entkommt, Phœbus wird für tot gehalten. Quasimodo rettet die des Mordes an Phobus verdächtigte Esmeralda vor der wütenden Menge und der Justiz und flieht mit ihr in sein Zimmer in Notre-Dame. Frollo spürt sie dort auf. Es kommt zu einer dramatischen Auseinandersetzung. Frollo wird in die Flucht geschlagen. Er kehrt jedoch zurück, narkotisiert Quasimodo und übergibt Esmeralda den Bogenschützen. Aus der Narkose erwacht, schwört Quasimodo, den Entführer zu töten. Er stürzt Frollo von einem der Türme von Notre-Dame. Es folgt die Apotheose: Quasimodo und Esmeralda finden sich im Tod vereint. ${ }^{11}$

Richtete sich die Vorstellung von Fremdem/Heroischem im Tanz bis in die 1830er Jahre noch nach traditionellen theatralen Regeln, durch den Aktaufbau ebenso wie durch die Ausgestaltung der Rollenfiguren, so scheint mit den 1840er/1850er Jahren die Konzentration auf die Verknüpfungsmöglichkeiten von Alterität und Heldischem durch Körperdarstellung und Tanzen zuzunehmen. Henri Justamants Version ist vor allem deshalb interessant, weil die mise en scène notiert wurde. Anders als Ballette, die sich ,nur' über verbalschriftliche und/oder vor allem gedruckte Libretti tradieren, geben die Regie- wie Choreografiemanuskripte einen detaillierten Einblick in das Geschehen. Die Libretti dienten in der Regel als Information oder auch Souvenir für den Zuschauer, das heißt, dass Tänze, die ja in den jeweiligen Aufführungen zu sehen waren, gänzlich fehlen oder nur gelegentlich erwähnt oder dramaturgisch begründet werden. Die zusätzlichen Informationen, welche die livrets de mise en scène bieten, betreffen nun auch die Gestaltung der Tänze und sie verdeutlichen den Aufbau, die Dynamik des Balletts, die Entfaltung oder Stillstellung der Interaktionen. Mit Verbalbeschreibungen und Strichfiguren dokumentiert Justamant das Geschehen als dramatisierten Dialog von pantomimischer ,Rede` und ,Gegenrede`, zudem zeichnet er die tänzerischen Aktionen der Darsteller (ihre Schritte, Haltungen und Bodenwege) auf. ${ }^{12}$

11 Justamants Ballett orientiert sich weitgehend am Roman Notre-Dame de Paris von Victor Hugo, indem es dem Handlungsablauf des Ersten und Zweiten Buchs, Kapitel, I-IV beziehungsweise dem Elften Buch, Kapitel IV folgt. Spezifisch zum Vergleich von Justamants Version und dem Roman von Victor Hugo siehe Gabi Vettermann: Bilder des Fremden. In: Claudia Jeschke [et al.]: Interaktion und Rhythmus. Zur Modellierung von Fremdheit im Tanztheater des 19. Jahrbunderts. München 2010, 369-388.

$12 \mathrm{Zu}$ Justamants Notierungsmethode vgl. Claudia Jeschke: Inszenierung und Verschriftung. Zu Aspekten der Choreographie und Choreo-Graphie im 19. Jahrhundert. In: Katharina Keim 
Wie in Danina, oder Joko [...] wird auch hier das fremde Paar, die Außenseiter, im Titel genannt, nämlich Quasimodo, der Glöckner, und La Bohémienne, Esmeralda. Das Fremde ist allerdings von Beginn an - Victor Hugo folgend - geografisch und sozial weniger präzise beschrieben als in Danina, oder Joko[...]. Die Handelnden sind nicht in der Kolonialgeschichte, sondern in der europäischen Geschichte verankert. Der Vergleich zwischen Danina, oder Joko [...] und Quasimodo ou La Bohémienne in Bezug auf die Provenienz der Schauplätze und Figuren (Brasilien, Kolonialzeit - Europa, Mittelalter) zeigt, dass in Quasimodo ou La Bohémienne fremder Raum oder Zeit bestenfalls zum Lokalkolorit beitragen, aber nicht, wie teilweise in Danina, oder Joko [...], die Handlungsmöglichkeiten oder Entwicklungen bestimmen. Und bei der Verbindung von Heroischem und Fremden ergibt sich kaum je die Notwendigkeit der Argumentation, vielmehr übernimmt die Choreografie dramaturgische Aufgaben, indem sie das Handlungspotenzial in Bildräumen kristallisiert. ${ }^{13}$ Dies geschieht mittels der spektakulären Konstellation der amorphen Figur des Glöckners von Notre-Dame und der Zigeunerin Esmeralda.

Quasimodo sticht ähnlich wie Joko vom Beginn seines Erscheinens auf der Bühne durch seine körperliche Andersartigkeit oder Fremdheit hervor: „C'est Quasimodo, ayant une tête hérissée de cheveux roux, une bosse entre les deux épaules, des jambes en croissant, de larges pieds, et und allure redoutable de vigueur et d'agilité." 14 Schnelle Bewegungen und Wendungen kennzeichnen seine im livret de mise en scène dokumentierten Aktionen. ${ }^{15}$ Monströses Aussehen und deshalb (zu erwartender) schwerfälliger Habitus verbinden sich mit spontanen Aktionen und Reaktionen. ${ }^{16}$ Quasimodo steht wie der anthropomorphe Joko in der Tradition der Groteske; ${ }^{17}$ im Unterschied zu diesem ist er jedoch kein dramatur-

[et al.] (Hg.): Theater obne Grenzen. Festschrift für Hans-Peter Bayerdörfer zum 65. Geburtstag (Münchener Universitätsschriften/Theaterwissenschaft; 1), München 2003, 256-265.

13 In Justamants Quasimodo wird die a-lineare Dramaturgie durch die Gliederung des Balletts in situative Bilder unterstützt. Diese tableaux, die jeweils verschiedene Schauplätze (reale Orte in Paris) zeigen, lassen die Generierung von Einzelnen, von Helden von Beginn an porös erscheinen oder eröffnen umgekehrt Freiräume, indem sie diese als Geschehen, im Geschehen zulassen oder indem sie sie selbst als ganz konkrete Bühnenräume zu Handelnden machen. Das zeigt sich besonders am Beginn und am Endes Balletts. Im Vergleich mit den anderen, auch den bekannteren zeitgenössischen Ballettfassungen von Notre Dame de Paris, vor allem denen von und nach Jules Perrot (London 1844), erscheint Henri Justamants Version weniger dramatisch denn als Mischung von Melodram und Revue.

14 Quasimodo ou La Bobémienne, Libretto im Regiebuch (Anm. 10), 3. Vgl. die nahezu identische Formulierung bei Victor Hugo: Jeschke: Interaktion und Rhythmus (Anm. 1), 223, Anm. 77.

15 Nicole Haitzinger: Quasimodo oder das sublime Monster. In: Claudia Jeschke [et al.]: Interaktion und Rhythmus. Zur Modellierung von Fremdheit im Tanztheater des 19. Jabrbunderts. München 2010, 210-230.

16 Ebd.

17 Vor dem Hintergrund von Hugos Roman ist Quasimodo ebenso taub wie aus diesem Grund stumm, und der Figur liegt möglicherweise auch eine real-existente historische Figur zugrunde. Der Glöckner aus Quasimodo ou La Bohémienne dürfte also nicht nur in der TanzTheater-Tradition der Groteske zu suchen sein, sondern ebenso im Alltag von Hugo, Justa- 
gisch agierender Spielmacher sondern eine amorphe, choreografisch konturierte Figur, deren kämpferischer, gleichzeitig kontrollierter - heroisch affiner? - Bewegungsduktus seinem Aussehen zu widersprechen scheint.

Quasimodos Erscheinung und Auftreten benötigen die Präsenz anderer Rollenfiguren, um identifizierbar zu sein, vor allem die der Bohémienne. Seine Mitspieler und die Masse akzeptieren ihn trotz seiner physischen Deformationen und eingeschränkten Bewegungsfähigkeit, ebenso wie sie sich Esmeralda wegen ihrer Schönheit und Tanzkunst zuwenden. Zum Beispiel tanzt die Bohémienne bei ihrem ersten Auftritt einen Bolero, einen spanischen, in Justamants Fassung virtuosen und langen Tanz, der die Tänzerin als handwerklich hervorragende Künstlerin zeigt wie er auch - durch die Wahl eben dieses traditionellen und interaktiv, emotiv aufgeladenen Nationaltanzes - die Rollenfigur als verführerisch und gleichermaßen beherrscht charakterisiert. ${ }^{18}$

Die Handlung wird nicht länger, wie traditionell üblich, von Protagonist und Antagonist getragen, sondern generiert sich durch Quasimodos Physis und Esmeraldas Tanz - die Choreografie befördert also die Story und nicht umgekehrt: Zwei äußerlich gegensätzliche (und sich bewegungsmäßig ergänzende) Figuren stören die überlieferte ästhetische Harmonie des Tanztheaters und sie de- und rekonstruieren, indem sie als konträres Paar fungieren, die glaubwürdige Entwicklung der Handlung. Ihre ästhetischen wie dynamischen Verschiedenheiten fungieren als durchsichtige, amorphe Rollenfolien innerhalb der Bild-Räume des Geschehens Folien, die gegeneinander verschoben werden können und so das Potenzial haben, die Tradition des Tanzes ebenso zu bedienen wie sie zu konterkarieren.

In den beiden Schlussbildern wird das Fazit der vorausgegangenen Story präsentiert, inszeniert. Quasimodo und Esmeralda zeigen alle Facetten ihrer Figur und ihre Interaktionen stehen argumentationslos im Raum, sie ergeben weniger dramaturgischen Sinn als dass sie - als choreografierter Entwurf - auf die Möglichkeitsdimension von sinnlicher Erfahrung verweisen. Das 5. Tableau spielt in der Kammer Quasimodos in Notre-Dame. Der Schauplatz befindet sich auf der Bühne in normalem Sichtabstand zum Publikum. Der Vorhang hebt sich, die Zuschauer sehen den missgestalteten Glöckner mit Esmeralda im Arm, die er vor der Verfolgung der Menge gerettet hat. Die folgende Szene zeigt die ebenso hingebungsvolle wie aussichtslose Liebe des Außenseiterpaares. Zeitnah wird der Bühnenraum durch die hektischen Interaktionen der weiteren am Drama Beteiligten in Atem gehalten. Die Dynamik der Szene bestimmt Quasimodo: Er macht sich zum Agen-

mant et cetera. Zur realen Figur des Quasimodo vgl. die Entdeckung von Adrien Glew: Tate Press Office: Papers in Tate Archive may provide clue to identity of Hugo's Quasimodo, (Pressemitteilung vom 15. August 2010), http://www.tate.org.uk/about/press-office/pressreleases/papers-tate-archive-may-provide-clue-identity-hugos-quasimodo, 29. März 2016.

18 Claudia Jeschke: Repertoire fremder Tänze. In: Claudia Jeschke [et al.]: Interaktion und Rhythmus. Zur Modellierung von Fremdheit im Tanztheater des 19. Jabrbunderts. München 2010, 81-120. 
ten Esmeraldas, indem er eindrucksvoll und vehement seine andersartige Körperlichkeit einsetzt. ${ }^{19}$

Mit minimaler Aktion hingegen spielt das 6. Bild auf den Türmen von NotreDame, die Theaterzuschauer betrachten das Geschehen aus weiter Ferne. Das Bild zeigt den senkrecht in die Tiefe stürzenden Don Claude und den Glöckner, der in einer Verlängerung der Senkrechten bewegungslos auf dem Turm steht (Abb., linke Seite). Auch das nächste Tableau vermittelt Stillstellung: Quasimodo befindet sich im Himmel, zu Füßen Esmeraldas, die ihre Arme um ihn schlingt (Abb., rechte Seite). Besonders die Nutzung der Bühnensenkrechten durch den Sturz erscheint als probates wie äußerst dramatisches bild- wie bewegungsorientiertes Mittel, den Blick auf die heroische Substanz der jeweiligen Persönlichkeiten zu lenken. Die Wechsel der Räume, der inhaltlichen und visuellen Brennpunkte des Geschehens, der Wechsel der visualisierten Perspektiven auf das Geschehen erklären nicht, sie lassen sich vielmehr als spektakuläres Display theatraler Effekte und Möglichkeiten interpretieren - ebenso wie die Verbindung von Heroischem mit einem befremdlichen amorphen Körper im Zusammenspiel mit einem weiteren fremden Körper. Spektakularität bedeutet in Justamants Quasimodo-Inszenierung vor allem die offene Verhandlung des sinnlich wahrnehmbaren Fazits vehementer innerer Reibung fremd-heroischer Figuren, die nun szenisch und bewegungsorientiert geäußert wird. Und damit das Möglichkeitsspektrum des Theatralen reflektiert.

\section{Der Traum vom Un-Heroischen:}

\section{La Péri $(1842 / 1843)^{20}$}

Kurz zum Inhalt des Balletts: Der Dichter Achmet versetzt sich mittels Opium in einen Rauschzustand, in dem er den Péris und ihrer Königin begegnet. Die Idee von Himmel und Erde verselbstständigt sich (scheinbar), so dass sich ästhetische Vorstellung und Umsetzung in die szenische Realität verknüpfen. Die Königin, La Péri, verliebt sich in den Dichter. Um mit ihm zusammen zu sein, inkarniert sie in die auf der Flucht (aus dem Harem des Paschas) erschossene Sklavin Léila; sie verwandelt sich also in eine irdische Frau. Im Laufe der Proben zum Ballett wurden zwei Möglichkeiten des Schlusses erwogen. Gautier konzipierte das unglückli-

19 Vgl. Quasimodo ou La Bobémienne, Libretto im Regiebuch (Anm. 10), 7, 8: Quasimodo begehrt auf gegen seinen Pflegevater, als dieser Esmeralda erneut zu rauben versucht: „il se jette sur le ravisseur, lui pose un genou sur la poitrine et lève un coutelas sur sa tête" (7), und als Don Claude ihn schließlich als seinen Diener erkennt und Gehorsam fordert, antwortet er „avec énergie qu'il restera, et qu'avant qu'on touche à la jeune fille' il faut qu'on le tue. Il lui présente le couteau“ (8).

$20 \mathrm{Zu}$ den Fassungen vgl. Gabi Vettermann: Fallbeispiele. Eine Episode: Esmeralda, La Esmeralda, La Zingara ... tanzt, oder: Tänze einer Ausstellung des Fremden. In: Claudia Jeschke [et al.]: Interaktion und Rhythmus. Zur Modellierung von Fremdheit im Tanztheater des 19. Jahrbunderts. München 2010, 369-388. 


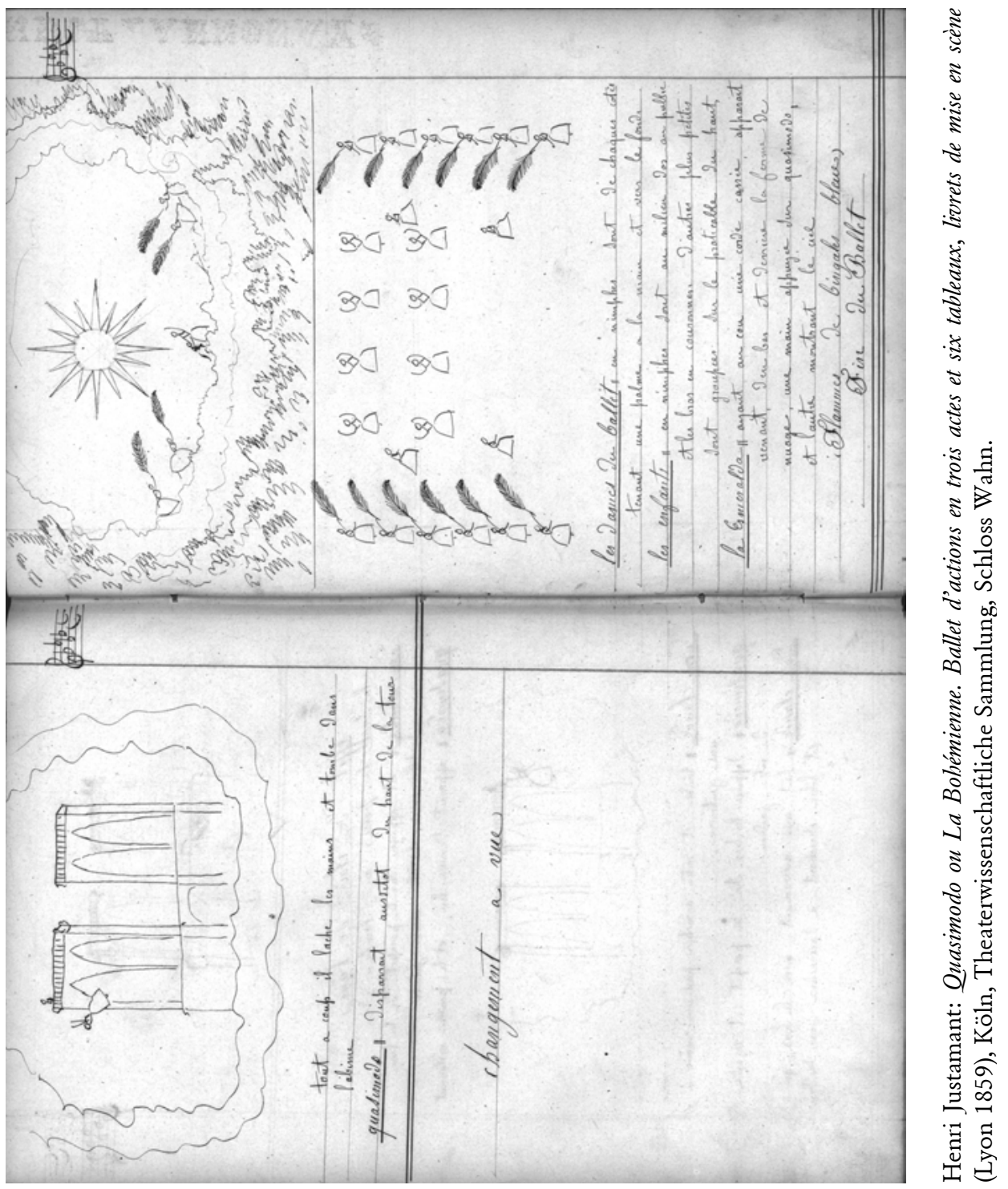


che Ende: Der Pascha nimmt Rache, Achmet wird, inspiriert durch Alexandre Decamps' Gemälde Le Supplice des crochets, hingerichtet. Kurz vor der Premiere wurde der unglückliche durch einen glücklichen Schluss ersetzt: durch die Apotheose von Achmet und Péri.

Wie Théophile Gautiers Erfolgsballett Giselle folgt auch La Péri einer zweiaktigen Struktur. Der Librettist legt also keinen Wert auf eine überlieferte dramatische Entwicklung und demgemäß auf sie bestimmende Schauplätze. Vielmehr stellt er sich alle Schlüsselstellen des Balletts als Tanz vor: unter anderem auch die Flucht der Péri über die Dächer beziehungsweise ihre Inkarnation in eine Sterbliche und den pas de l'abeille. La Péri ist, so Gautier, der Traum der Erde vom Himmel und der Traum des Himmels von der Erde. Das Ballett thematisiert die Überführung einer ästhetischen Vorstellung in eine von heroisch agierenden Fremden getragene Tanz-Handlung. ${ }^{21}$

Im Unterschied zum heimlichen Paar und zum konträren Paar der vorangegangen Beispiele handelt es sich in La Péri um ein Traum-Paar. Dieses Paar hat das Potenzial zur Realisierung ihrer Beziehung - dadurch, dass Achmet Péri in Léila wieder-erkennt. Dazu bedarf es des Muts zur Transformation, nicht nur von Péris Seite (durch ihre Verwandlung zu Léila), sondern ebenso von Achmets Seite, dessen Traum von der Königin der Péris durch die Begegnung mit einer Sterblichen, Leila, auf dem Spiel steht.

Die Verwandlung der Péri zu Léila erfolgt, als eine Sklavin dem Pascha entflieht, von seinen Dienern verfolgt und erschossen wird. Blitzschnell, unterstützt vermutlich durch Lichteffekte, inkarniert La Péri in die Getötete, legt alle ihre Insignien $\mathrm{ab}$ und wird sterblich. In dieser Form sieht sie ihre Chance, sich Achmet zu nähern. Ein (scheinbar) zufälliger theatraler Moment kreiert Abwesenheit von Interaktion und unbedingte tänzerische Stillstellung - momenthafte Löschungen, in denen Eigenes, Fremdes, Heroisches nicht ausgestellt oder verhandelt werden, in denen sich jedoch das folgende Geschehen als Intention der Péri (und aufgrund der Paarkonstellation indirekt auch Achmets) als Subtext, als Programm ankündigt: Es bedarf der Fantasie, um die Annäherung an das Heroisch-Fremde zu ermöglichen.

Als Dreh- und Angelpunkt des Wieder-Erkennens durch Achmet funktioniert der pas de l'abeille, der Bienentanz. Er ist in allen Versionen des Librettos als Krönung eines Festes und damit zunächst als fremd-tänzerische (physische, energetische) Charakterisierung der Protagonistin konzipiert. Darüber hinaus präsentiert dieser Tanz eine choreografisch besondere Form der Kommunikation, die pantomimische Auseinandersetzung mit einer gefährlichen Biene (als Metapher für

21 Persönliches Anliegen wie die in der Forschung mehrfach attestierte Experimentierfreudigkeit und Radikalität zeichnen das Ballett von Beginn seiner Entstehung aus. Vgl. auch Andrew Gann: La genèse de la Péri. In: Théophile Gautier: L'art et l'artiste. Actes du colloque organisé en 1982 par la Société Théophile Gautier, Bd. 1. Montpellier 1982, 207-220. 
Achmet?22), und entwickelt dadurch eine narrative Aufladung der tanztheatral kinästhetischen Wirkung ${ }^{23}$.

Elle s'avance au milieu du théâtre, se débarrasse de son manteau et s'apprête à danser un pas national connu au Caire sous le nom de Pas de l'abeille. - La danseuse cueille un rose: l'insecte irrité sort en bourdonnant du calice de la fleur et poursuit l'imprudente, qui tâche de l'écraser tantôt entre ses mains, tantôt sous son pied. - L'abeille va être prise: un mouchoir dont Léila relève le coin avec précaution rend ses ailes inutiles. Mais quoi! elle s'est échappée, et, plus irritée que jamais, elle se glisse dans le corsage de la danseuse, qui la cherche dans les plis de sa veste, dont elle se débarrasse; la lutte continue, l'abeille bourdonne, la jeune fille tourbillonne, augmentant toujours la vivacité de sa danse. La ceinture va bientôt rejoindre la veste, et Léila, dans le costume le plus léger, en simple jupe de gaze, continue ses évolutions éblouissantes, et finit, éperdue, haletante, par aller chercher un abri sous la pelisse d'Achmet, qui, ravi d'admiration, s'incline amoureusement vers elle, et lui couvre, à la mode orientale, le front et la poitrine des pièces d'or. Leila reçoit modestement les félicitations des assistants et lorsque les femmes se retirent, Achmet la fait rester auprès de lui. ${ }^{24}$

Die unterschiedliche Platzierung des Tanzes in den verschiedenen Fassungen des Librettos, die zum Teil während des Probenprozesses entstanden, zeigt, dass sich Transformation und Wieder-Erkennen schwer umsetzen ließen. Auch ein traditioneller argumentativer Vorschlag wie die Einführung einer Antagonistin, der bisherigen Favoritin Achmets, die sich nicht so einfach von ihrer Stellung vertreiben lassen wollte und folglich Péri/Léila nach dem Leben trachtete, konnte das dramaturgische beziehungsweise choreografische Problem nicht lösen. Die Verwandlung der Péri wurde auf diese Weise immer nur indirekt angesprochen. Und als bloßer Subtext taugte sie zwar zur spektakulären Vermittlung von Fremdem, aber nicht von Heroischem. Die Idee des Traums, des Traums von einem Paar, funktionierte erst, als der pas de l'abeille und das Wieder-Erkennen entkoppelt wurden. Die Freistellung des pas de l'abeille zum narrativen wie virtuosen, verführerischen Schaustück machte es möglich, die Frage nach der Identität von Léila oder der Verwandlung der Péri nicht mehr zu stellen. Achmet akzeptiert fraglos die Wirkkraft, welche die tänzerischen Aktionen auf ihn haben - der pas de l'abeille impliziert so die Möglichkeit zur Interaktion von Péri/Léila und Achmet als Paar. Die Tatsache, dass der Schluss durch Apotheose oder Hinrichtung (Le Supplice des crochets) ge-

22 Vgl. Nicole Haitzinger: Fallbeispiele. Pas de l'Abeille und Cachucha - Auftritte von Frauen als andere Fremde. In: Claudia Jeschke [et al.]: Interaktion und Rhythmus. Zur Modellierung von Fremdheit im Tanztheater des 19. Jahrhunderts. München 2010, 199-209, hier 208.

23 Die Verwendung des Begriffs ,Kinästhesie‘ folgt der Definition von Carrie Noland: Agency and Embodiment: Performing Gestures/Producing Culture. Cambridge, MA [u.a.] 2009, 9: „the term ,kinesthesia' refers to the sensations of movement transmitted to the mind from the nerves of the muscular, tendinous, and articular systems." Die Übertragung geschieht nicht nur propriozeptiv, sondern auch kommunikativ, das heißt, sie betrifft die Interaktionen mit den Mit-Tänzern auf der Bühne sowie den anwesenden Zuschauern.

24 Théophile Gautier: CEuvres complètes, Section III: Théâtre et ballets, hg. von Claudine Lacoste-Veysseyre und Hélène Laplace-Claverie. Paris 2003, 611. 
plant war beziehungsweise in einer Fassung auch stattfand, verweist einmal mehr auf das verstörende Potenzial der heroisch-affinen Metamorphosen.

Die Interpretationen der performatorischen Dimension von Libretti und Inszenierungsnotaten markieren je eigene szenische Kollusionen und Kollisionen von literaler Narrativik, tänzerischer Narration, Inszenierung, Bedeutung und kinästhetischer Wirkung. Die unterschiedlichen Konstellationen des Heroischen und Fremden - so zeigen die Fallbeispiele - fordern und fördern die Entwicklung tanztheatraler Frei- und Spiel-Räume für das Heroisch-Fremde, indem sie herkömmliche, dramaturgisch und/oder choreografisch literalisierte Schlüsselsituationen (hier beispielhaft: Interaktionen und Tänze) ereignishaft fragmentieren und de- beziehungsweise reformieren. Die Ereignishaftigkeit bezieht sich in Danina, oder Joko [...] auf die in den verschiedenen Librettofassungen vorgestellten Entwürfe von Heroischem in kolonialen und antropomorphen Fremden und diverse Experimente mit deren vor allem dramaturgischer Praktikabilität. In Quasimodo ou La Bohémienne wird Handlung weniger linearisiert als in (bewegte) Bilder gefasst: Die phänomenologisch-psychologische Verdichtung der tanzend-handelnden Fremden/Anderen rhythmisiert - bildhaft dramatisierend - das Geschehen und öffnet es so dem Entwurf eines bislang unbekannten wie unerwarteten Heroischen. Und die Libretti und der Probenprozess zu La Péri präsentieren sowohl die Interaktionen des fremd-heroischen Paares wie auch die Tänze jenseits, ja in Umkehrung von herkömmlicher dramaturgischer Narrativik und tänzerischer Narration: Die Verwandlung der Péri in Léila erfolgt argumentationslos chimärisch während eines kurzen Moments der Stillstellung. Die Positionierung wie provokante Choreografie des pas de l'abeille impliziert Handlungsoptionen für das Paar, die sich der szenischen Realisierung durch Apotheose entziehen.

In der Zusammenschau der drei Fallbeispiele entfalten die morphologischen Verkörperungen und dadurch bedingten Verschiebungen von heroisch-fremd profilierten Figurationen ein weites Spektrum heldisch-affiner Eigenschaften und Handlungen, welche die Entwurfspotenziale tanztheatraler Praxen und Inszenierungen im 19. Jahrhundert von der literalen Argumentation zur ereignishaften Spektakularität erweitern.

\section{Abbildungsnachweis}

(C) Theaterwissenschaftliche Sammlung, Schloss Wahn, Köln. Für die Abdruckgenehmigung bedanken wir uns bei Hedwig Müller. 